\title{
Uretrocistopexia suprapúbica tipo Burch por laparoscopia (Estudio piloto)
}

\author{
Jorge Andrés Rubio Romero*; Jacinto Sánchez Angarita**; Antonio Lomanto Morán***; Pío Iván Gómez Sánchez****; \\ Liliana Arango Rodríguez ${ }^{* * * *}$
}

\section{RESUMEN}

OBJETIVO: Describir la técnica por laparoscopia de la Uretrocistopexia Suprapúbica (UCSP) tipo Burch para el tratamiento de la Incontinencia Urinaria de Esfuerzo Verdadera (IUE) y evaluar su eficacia a corto plazo.

METODOLOGIA: Se realizó un estudio prospectivo, descriptivo, de serie de casos en un centro de atención terciaria en Santafé de Bogotá. Se evaluaron 17 pacientes con IUE sometidas a tratamiento quirúrgico de UCSP tipo Burch por laparoscopia, evaluando la persistencia y/o recurrencia de la IUE, las complicaciones intra y post-operatorias, tiempo quirúrgico, sangrado, requerimiento de analgésico y la estancia hospitalaria.

RESULTADOS: El abordaje por laparoscopia presenta una eficacia durante el tiempo de seguimiento del $94.2 \%$, con un tiempo quirúrgico promedio de $\mathbf{1 2 0 . 3}$ min y mayor presencia de complicaciones intra-operatorias. El requerimiento de analgésicos postoperatorios fue de $\mathbf{2 8 2 . 3} \mathbf{m g s}$ y la estancia hospitalaria de $\mathbf{3 . 5}$ días.

CONCLUSIONES: EI abordaje por laparoscopia constituye una alternativa promisoria para la corrección de la IUE pero puede producir complicaciones importantes y un tiempo operatorio prolongado durante la fase de aprendizaje de la técnica, situación que debe mejorar al aumentar la experiencia en la realización de la misma en pacientes seleccionadas. Debe iniciarse el estudio comparativo, aleatorizado y controlado de la técnica por laparoscopia con seguimiento a largo plazo contra la técnica abierta tradicional, en la que se cuenta con gran experiencia y resultados favorables conocidos.

PALABRAS CLAVES: Incontinencia urinaria de esfuerzo, uretrocistopexia suprapúbica tipo Burch, laparoscopia.

\section{SUMMARY}

OBJECTIVE: To describe laparoscopic suprapubic Burch uretrocistopexy technique and effectiveness at short term for Stress Urinary Incontinence (SUI).

METHODS: Prospective, descriptive case series study at a terciary attention Centre. 17 women with SUI were treatted with a laparoscopic Burch procedure. Study variables were recurrence and/or persistence of SUI after surgery, trans and post-operative morbidity, operative time, blood loss, post-operative pain and hospital stay for each group.

RESULTS: Laporoscopic Burch procedure has a short term effectiveness of $\mathbf{9 4 . 2} \%$, longer operative time (120.3 min) and greater number of trans-operative complications. Post-operative analgesics requirements were 282.3 mgs and hospital stay was 3.5 days.

CONCLUSIONS: Laparoscopic Uretrocistopexy is a promising technique for SUI treatment but it may carry severe complications and a longer operative time during the learning phase of the technique. This situation will vary as experience is gained as the laparoscopic Burch procedure is performed on selected patients. A comparative, controlled and randomized study with long term follow-up is required.

KEY WORDS: Stress urinary incontinence, suprapubic Burch uretrocistopexy, laparoscopy.

Residente III Año. Ginecología y Obstetricia.

** Profesor Asociado. Ginecología y Obstetricia. Clínica de Incontinencia Urinaria.

*** Profesor Asociado. Ginecología y Obstetricia. Clínica de Incontinencia Urinaria.

Profesor Asociado. Asesor Unidad de Cirugía Ambulatoria.

***** Gineco-Obstetra. Unidad de Cirugía Ambulatoria.

\section{Introducción}

La incontinencia urinaria de esfuerzo verdadera o pura (IUE) es la salida involuntaria de orina a través de la uretra, en ausencia de contracciones no inhibidas del detrusor, como consecuencia del aumento súbito de la presión intra-abdominal, debido a la pérdida del soporte anatómico del cuello vesical y la uretra proximal, por 
relajación de los ligamentos uretro-pélvicos y de los músculos elevadores del ano (1).

La incontinencia urinaria de esfuerzo es un problema frecuente, que afecta entre el $5-10 \%$ de las mujeres a cualquier edad (2) llegando a afectar cerca de 10 millones de mujeres norteamericanas, según el National Institute of Health, con un costo financiero enorme calculado para 1988 en cerca de 10.3 billones de dólares al año. En el Instituto Materno Infantil, se ha calculado que la IUE afecta cerca del $5.1 \%$ de las mujeres que asisten a consulta externa de Ginecología al año, y dicha frecuencia aumenta progresivamente con la edad (6). Así, la incontinencia urinaria de esfuerzo constituye un problema social y de salud por el cual la mujer consulta al médico en busca de soluciones prontas, económicas y efectivas.

Múltiples tratamientos han sido implementados para la corrección de esta anomalía, que tiene un gran impacto sobre la vida social y el bienestar de las pacientes. Se ha intentado desde el tratamiento médico con fisioterapia para el fortalecimiento del piso pélvico (ejercicios de Kegel), el uso de conos vaginales de peso creciente, medicamentos a-adrenérgicos, el uso de estrógenos en las pacientes postmenopáusicas y la estimulación eléctrica del piso pélvico, con resultados variables en efectividad.

Varias técnicas quirúrgicas han sido desarrolladas para la corrección de la incontinencia urinaria de esfuerzo y tienen como principal objetivo la colocación de la uretra proximal y el cuello vesical en una posición alta, retropúbica y bien sostenida para transmitir la presión intra-abdominal en forma simultánea tanto al cuerpo como al cuello vesical. Estas técnicas están descritas por vía vaginal, suprapúbica abierta y percutánea para lograr estos objetivos (2).

La evaluación de los métodos de corrección de la incontinencia ha demostrado que las técnicas de suspensión suprapúbicas, especialmente la descrita por Burch (3), son las de mayor efectividad con resultados exitosos entre el $80-90 \%$ de los casos en estudios de seguimiento a largo plazo, pero con el inconveniente de una mayor morbilidad asociada al procedimiento, comparadas con técnicas vaginales o percutáneas. En el lnstituto Materno Infantil, se reporta una eficacia del $94 \%$ en un estudio de seguimiento a cinco años (6).

En 1991, Vancaillie y Schuessler describieron la uretrocistopexia suprapúbica por vía laparoscópica (4), abriendo la posibilidad de realizar la corrección de la incontinencia urinaria de esfuerzo con, al menos desde el punto de vista teórico, una disminución de la morbilidad asociada al procedimiento y una reintegración pronta a las actividades diarias de las pacientes.

Dado que en la actualidad ya están descritas y se practican estas técnicas de corrección por la vía laparoscópica, existe una gran preocupación por la eficacia de esta nueva aproximación comparada con la técnica quirúrgica tradicional, aunque los actores afirman respetar la técnica original en el momento de realizar la aproximación por laparoscopia. Dada la innovación y las dificultades propias del campo operatorio laparoscópico, esta afirmación corre el riesgo de no ser del todo cierta, y por ende, hace ineludible la necesidad de realizar estudios comparativos que evaluúen la eficacia y la morbilidad de las técnicas laparoscópicas comparadas con las técnicas abiertas tradicionales tanto abdominales como vaginales, como la afirman Dorsey y Cundiff (7).

Las ventajas teóricas del abordaje por laparoscopia de una técnica reconocida, efectiva y segura como la técnica de Burch son:

- una herida quirúrgica pequeña, disminución del dolor post-operatorio, menor requerimiento de analgésicos (8-9), disminución del tiempo de cicatrización y un resultado cosmético mejor. Esto a su vez permite una recuperación más rápida y un tiempo de convalescencia menor.

- La mejor visualización de las estructuras anatómicas permite una disección roma más fina, atraumática, menor edema post-operatorio, menor trauma y reacción inflamatoria de los tejidos manipulados, y el control minucioso del sangrado durante el transoperotorio, disminuyendo las pérdidas hemáticas. Todo lo anterior, permite una disminución importante en la morbilidad intra y postoperatoria de la corrección de la IUE (8-10).

- La recuperación más rápida permite disminuir el tiempo de hospitalización de las pacientes y el retorno a la actividad productiva en forma más temprana, lo cual se refleja en menores costos de atención en salud para las pacientes con IUE.

La técnica laparoscópica tendría como inconveniente un mayor tiempo quirúrgico (8-9), dependiente de la curva de aprendizaje de la técnica, incremento en el costo del procedimiento, exposición a dosis mayores de anestésicos y aumento del riesgo quirúrgico. Además, un mayor riesgo de complicaciones dado por el momento ciego de la misma, cuando la posibilidad de perforación de una vísera intra-abdominal y de otros órganos, incluyendo estructuras vasculares retroperitoneales, es mayor. Por supuesto, el buen uso de la técnica, su conocimiento y el sentido de la responsabilidad permiten disminuir al máximo este riesgo, además de la posibilidad de un abordaje completamente extraperitoneal, que disminuye notoriamente el riesgo de estas complicaciones.

Estudios recientes con seguimiento a corto plazo, en los que se ha utilizado la técnica laparoscópica para la corrección de la IUE, han demostrado efectividad entre el 78 y el $94 \%$, en un período de 12 meses, lo que parece hacer comparable esta técnica con el método abierto tradicional. Sin embargo, hasta el momento no se han reportado estudios clínicos controlados que confirmen esta hipótesis tanto a mediano como a largo plazo (7-10, 12).

Mediante este estudio, se pretende describir la técnica y establecer la persistencia y recurrencia de la IUE luego de la uretrocistopexia suprapúbica tipo Burch laparoscópica extraperitoneal a corto plazo, evaluar las complicaciones intra y post-operatorias de dicha técnica, establecer el tiempo quirúrgico, la cantidad estimada de sangrado y el requerimiento de analgésicos, para fomentar el aprendizaje de esta nueva técnica quirúrgica y el desarrollo tecnológico en el personal docente y en entrenamiento del Instituto Materno Infantil y la Universidad Nacional de Colombia, como parte inicial del estudio comparativo aleatorizado y controlado a largo plazo de la Clínica de Incontinencia Urinaria, comparando la efecti- 
vidad de los abordajes abierto tradicional y laparoscópico para la corrección de la IUE.

\section{Materiales y métodos}

Se realizó un estudio prospectivo, observacional, de serie de casos, descriptivo de una técnica quirúrgica, regido estrictamente por las consideraciones éticas establecidas en la declaración de Helsinski en 1964 y sus modificaciones de Japón de 1975 y de Venecia en 1988.

\section{Población de estudio}

Todas las pacientes con diagnóstico comprobado de incontinencia urinaria de esfuerzo pura, que consultaron a la clínica de Incontinencia Urinaria del Instituto Materno Infantil de la ciudad de Santafé de Bogotá, centro universitario de referencia, entre el 1o. de noviembire de 1996 hasta octubre 31 de 1997.

Se tomó una muestra secuencial de 17 pacientes, quienes luego de la firma del consentimiento informado, fueron asignadas al grupo de tratamiento por laparoscopia a criterio del docente investigador, según sus características de peso, índice de masa corporal (IMC) y la ausencia del antecedente de cirugía abdominal previa.

\section{Criterios de inclusión}

Se incluyeron las pacientes mayores de edad que consultaron directamente o fueron remitidas a la clínica de incontinencia urinaria del Instituto Materno Infantil, y que luego de su evaluación clínica, manifestaron libre y voluntariamente su deseo de ser tratadas para la corrección de su problema de incontinencia, en el período señalado.

\section{Criterios de exclusión}

Se excluyeron las pacientes con una o más de las siguientes condiciones:

- Incontinencia urinaria mixta.

- Cirugías para patología pélvica asociada. (Por ejemplo: histerectomía por miomatosis uterina, prolapso genital, cirugía para corrección de celes, etc.,)

- Otros procedimientos previos para la corrección quirúrgica de la IUE.

- Contraindicación anestésica ASA IV.

Las pacientes fueron valoradas en la consulta de incontinencia urinaria, en donde se les realizó la historia clínica y examen físico detallado en dicha clínica, incluyendo examen neurológico y pélvico bimanual. Además, se les realizó urocultivo, cistoscopia y urodinamia a todas las pacientes candidatas.

\section{Técnica quirúrgica}

Las pacientes fueron preparadas de la manera usual para cirugía de incontinencia abierta. Se les aplicó una dosis de antibiótico profiláctico al entrar en la sala de cirugía y se les tomó una muestra de sangre para determinar su hemoglobina y hematocrito. Todas las pacientes recibieron anestesia general. El procedimiento quirúrgico fue realizado por cirujanos certificados en el nivel de Expertos en cirugía laparoscópica.
Previa asepsia y antisepsia, con la paciente en posición de litotomía, se realizó cateterización vesical con sonda de Foley y se insufló el balón de la misma con 30 cc de Solución Salina normal al $0.9 \%$.

Se realizó una incisión infraumbilical transversa de un centímetro hasta la fascia de los rectos, disecando luego el espacio preperitoneal y desarrollando el espacio de Retzius mediante un balón inflable.

Luego de la introducción del laparoscopio por dicha incisión y dos puertos laterales para la instrumentación, se realizó disección roma del espacio vesical hasta la visualización de la fascia para-cervical y la pared vaginal anterior, colocando un punto a cada lado del cuello vesical sobre la fascia, llevándolo luego hasta el ligamento de Cooper y anudándolo mediante baja-nudos, elevando el cuello vesical con ayuda de los dedos desde la vagina.

Posteriormente, luego de revisar la hemostasia y la vejiga en busca de lesiones, se dejaron $10 \mathrm{cc}$ de xilocaína al $1 \%$ en el espacio extra-peritoneal, se retiraron los instrumentos y se cerró la piel de la manera acostumbrada.

A todas las pacientes se les realizó colporrafia posterior como complemento de su cirugía para el tratamiento de la incontinencia.

Terminado el procedimiento quirúrgico, las pacientes recibieron una dosis de IM de $75 \mathrm{mgs}$ de Diclofenac y luego de $50 \mathrm{mgs}$ vía oral, según los requerimientos subjetivos de cada paciente, dieta en las siguientes seis horas, momento en el cual se les tomó una nueva muestra de sangre para hemoglobina y hematocrito. Se retiró la sonda vesical a las 24 horas del procedimiento de no existir contraindicaciones para ello y una muestra para urocultivo, siempre y cuando el residuo post miccional fuera menor de $100 \mathrm{cc}$.

Las pacientes fueron dadas de alta según su evolución clínica previa evaluación anestésica y ginecológica y fueron evaluadas a los 8 días, al mes, a los tres, seis y doce meses luego del procedimiento, para el seguimiento de su evolución clínica luego de la cirugía.

\section{Desenlaces}

Los desenlaces tenidos en cuenta en el presente estudio fueron 1) persistencia de la IUE (reaparición de la sintomatologia de esfuerzo antes del primer año post-operatorio), 2) complicaciones intra-operatorias (lesión vesical, lesión vascular, lesión de vísera hueca, sangrado excesivo, necesidad de laparotomía para la técnica laparoscópica) y post-operatorias (infección de vías urinarias, hematuria, retención urinaria (residuo mayor de $100 \mathrm{cc}$ ), incontinencia urinaria de urgencia, hematoma o infección de herida o sitio quirúrgico), 3 ) tiempo quirúrgico (contabilizado desde la incisión hasta el cierre completo de la piel, excluyendo la colporrafia posterior), 4) sangrado intraoperatorio (determinado por la diferencia de concentración de Hemoglobina pre y post-operatorio) 5) requerimiento de analgésicos post-operatorios (cantidad de mgs de Diclofenac por vía oral durante la hospitalización) y 6) estancia hospitalaria. 


\section{Recolección, procesamiento y análisis de la información}

La información fue recolectada en forma manual en el formulario de recolección de datos diseñado para tal fin. El procesamiento de la información se realizó en forma manual y mediante el programa EPI Info 6.0 de la Oficina de epidemiología de la CDC de Atlanta, EUA. Las variables se analizaron en tablas y gráficas, las cuales se incluyen en los resultados.

\section{Resultados}

Se incluyeron 17 pacientes entre 32 y 57 años. La edad promedio del grupo fue de $43 \pm 6.6$ años. El peso promedio para el grupo fue de $57.6 \pm 8.6 \mathrm{Kgs}$ con un rango entre 43 y $79 \mathrm{kgs}$. El índice de masa corporal fue de $23.7 \pm 3.3 \mathrm{kgs} /$ $\mathrm{m}^{2}$ entre 17 y $30 \mathrm{Kgs} / \mathrm{m}^{2}$ (Tabla 1).

La duración promedio de la cirugía por abordaje laparoscópico fue de $120.3 \pm 48$ minutos, con un rango entre 60-210 minutos, incluyendo aquellas que por complicaciones terminaron en laparotomía.

La hemoglobina pre-operatoria fue de $14.8 \pm 1.15 \mathrm{Grs} /$ $\mathrm{dL}$ y la hemoglobina post-operatoria fue de $12.7 \pm 1.6 \mathrm{grs} /$ dL. El sangrado intra-operatorio, calculado a partir de la diferencia pre y post-operatoria de la concentración de la hemoglobina fue de $2.0 \pm 1.0 \mathrm{grs} / \mathrm{dl}$, que corresponde a una variación del $13.9 \%$ (Tabla 2 ).

Tabla 1

\section{URETROCISTOPEXIA SUPRAPUBICA TIPO BURCH POR LAPAROSCOPIA (ESTUDIO PILOTO)}

CARACTERISTICAS BIOMETRICAS
DE LAS PACIENTES

\begin{tabular}{|ll|}
\hline & Cirugía Laparoscópica \\
\hline Número Pacientes & 17 \\
Edad años & $43.0 \pm 6.6$ \\
Peso $(\mathrm{Kgs})$ & $57.6 \pm 8.6$ \\
Rango $(\mathrm{Kgs})$ & $43-79$ \\
IMC $\left(\mathrm{Kgs} / \mathrm{m}^{2}\right)$ & $23.7 \pm 3.3$ \\
\hline
\end{tabular}

IMC $=$ Indice de Masa Corporal.

Tabla 2

URETROCISTOPEXIA SUPRAPUBICA TIPO BURCH POR LAPAROSCOPIA (ESTUDIO PILOTO)

SANGRADO INTRAOPERATORIO

\begin{tabular}{|ll|}
\hline & Cirugía Laparoscópica \\
\hline Hb pre-operatoria & $14.8 \pm 1.15 \mathrm{Grs} / \mathrm{dL}$ \\
Hb Post-operatoria & $12.7 \pm 1.6 \mathrm{Grs} / \mathrm{dL}$ \\
Diferencia & $2.0 \pm 1.0 \mathrm{Grs} / \mathrm{dL}$ \\
Variación & $13.9 \%$ \\
\hline
\end{tabular}

De las 17 cirugías abordadas por laparoscopia, se presentaron complicaciones en cuatro (4) de ellas (23.4\%) por lo que terminaron en laparotomía; tres (3) por lesiones vesicales y una por sangrado excesivo (Tabla 3 ).

En el post-operatorio, cinco (5) de las 17 presentaron complicaciones $(29.4 \%)$. Dos (2) pacientes presentaron infección de vías urinarias, confirmadas por urocultivo, dos presentaron retención urinaria (11.7\%) y una paciente fue llevada a laparotomía cuatro días después de la cirugía, por una lesión vesical inadvertida durante la laparoscopia, realizándole cistorrafía y evolucionando sin complicaciones posteriores (Tabla 4).

Las pacientes requirieron un promedio de $282.3 \pm 123$ mgs de Diclofenac intrahospitalario y permanecieron hospitalizadas un promedio de 3.5 días, a excepción de la paciente con lesión vesical inadvertida quien permaneció hospitalizada 25 días.

El seguimiento promedio hasta el momento es de 3.0 meses. Durante el primer control post-operatorio, las 17 pacientes refirieron una adecuada continencia urinaria y una de ellas presentó infección de vías urinarias. Trece de las 17 pacientes han sido valoradas al tercer mes, sin encontrar complicaciones ni incontinencia. Seis pacientes han completado el sexto mes post-operatorio. Una de ellas presentó un episodio de infección de vías urinarias y otra refirió sintomatología de urgencia. Las restantes

Tabla 3

URETROCISTOPEXIA SUPRAPUBICA TIPO BURCH POR LAPAROSCOPIA (ESTUDIO PILOTO)

\begin{tabular}{|ll|}
\hline & Cirugía Laparoscópica \\
\hline Duración Promedio (min) & $120.3 \pm 48$ \\
Residuo post-operatorio (cc) & 58.9 \\
Analgésico (mgs) & 282.3 \\
Estancia (días) & 3.5 \\
\hline
\end{tabular}

Tabla 4

URETROCISTOPEXIA SUPRAPUBICA TIPO BURCH POR LAPAROSCOPIA (ESTUDIO PILOTO)

COMPLICACIONES INMEDIATAS

\begin{tabular}{|ll|}
\hline Complicaciones & Cirugía Laparoscópica \\
\hline Intra-operatorias (4/39) & $4 / 17$ pacientes \\
& Lesiones vesicales: 3 \\
& Laparotomía por Sangrado: 1 \\
Post-operatorias (10/39) & 5/17 pacientes \\
& IVU: 2 \\
& Retención: 2 \\
& Lesión Vesical: 1 \\
& $9 / 17$ \\
\hline
\end{tabular}

IVU $=$ Infección Vías Urinarias. 


\section{Gráfico 1 \\ URETROCISTOPEXIA SUPRAPUBICA TIPO BURCH POR LAPAROSCOPIA (ESTUDIO PILOTO)}

\section{COMPLICACIONES INMEDIATAS INTRAOPERATORIAS}

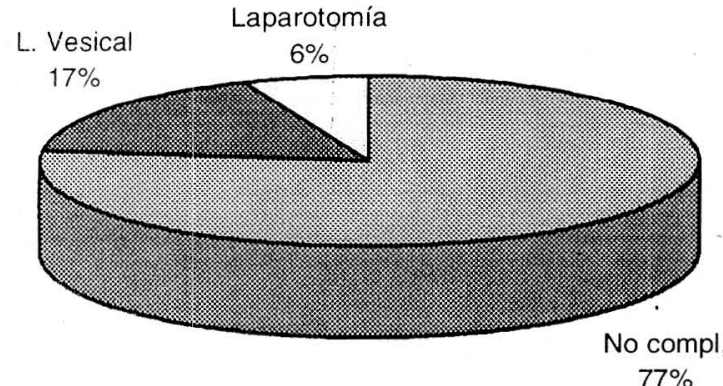

Gráfico 2

\section{URETROCISTOPEXIA SUPRAPUBICA TIPO BURCH} POR LAPAROSCOPIA (ESTUDIO PILOTO)

COMPLICACIONES INMEDIATAS POST-OPERATORIAS

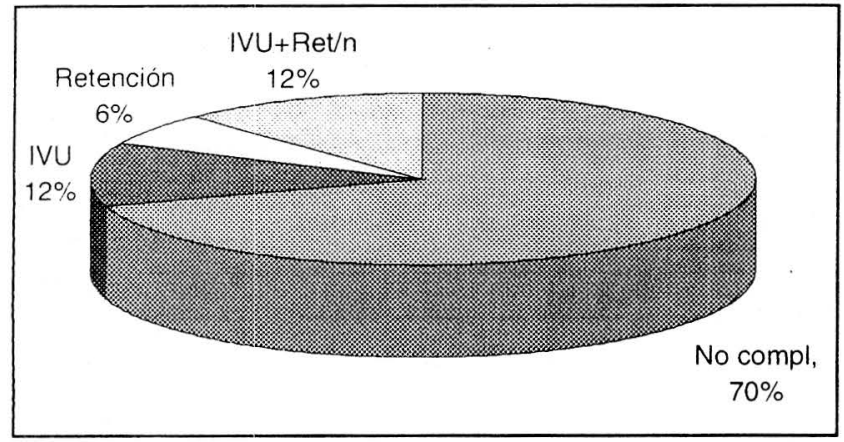

Tabla 5

\section{URETROCISTOPEXIA SUPRAPUBICA TIPO BURCH POR LAPAROSCOPIA (ESTUDIO PILOTO)}

SEGUIMIENTO

\begin{tabular}{|ll|}
\hline Seguimiento & $\begin{array}{l}\text { Cirugía Laparoscópica } \\
\text { Complicaciones }\end{array}$ \\
\hline $\begin{array}{l}\text { Primera semana } \\
\text { (39 pacientes) }\end{array}$ & IVU: 17 pacientes \\
Un mes & $0 / 17$ pacientes \\
(38 pacientes) & $0 / 13$ pacientes \\
$\begin{array}{l}\text { Tercer mes } \\
(28 \text { pacientes })\end{array}$ & $2 / 6$ pacientes \\
$\begin{array}{l}\text { Sexto mes } \\
\text { (17 pacientes) }\end{array}$ & IVU: 1 \\
Un año & Urgencia: 1 \\
(4 pacientes $)$ & $0 / 2$ pacientes \\
\hline Eficacia & $94.2 \%$ \\
\hline
\end{tabular}

IVU: Infección Vías Urinarias

Urgencia: Sintomatología de Urgencia. están asintomáticas. Dos pacientes han completado un año luego de su cirugía, se conservan continentes y no han presentado complicaciones. En total, el $94.2 \%$ de las pacientes no presenta incontinencia de esfuerzo o urgencia (Tabla 5).

\section{Discusión}

La técnica de Burch es actualmente reconocida como una de las más efectivas para el tratamiento de la incontinencia urinaria de esfuerzo. Estudios a 5 años demuestran tasas de efectividad entre $84-94 \%$ en grupos con experiencia $(5,16)$. Para la Clínica de Incontinencia Urinaria del Instituto Materno Infantil, la experiencia a cinco años de la uretrocistopexia suprapúbica es del $94 \%$ por la técnica abierta tradicional, a la que de rutina se le realiza Colporrafia Posterior.

Desde 1991, aparecen en la literatura series de casos de uretrocistopexia suprapúbica tipo Burch realizada por laparoscopia, con tasas de éxito similares a la tasa obtenida con la técnica abierta tradicional, ventajas desde el punto de vista operatorio, estético y de recuperación y tasas de complicaciones similares $(8-12,17,19)$.

El presente estudio fue diseñado para además de iniciar el aprendizaje y el desarrollo de la técnica laparoscópica de la Uretrocistopexia suprapúbica, evaluar la eficacia inicial del abordaje laparoscópico, para establecer si en nuestro medio, donde se cuenta con una amplia experiencia en la realización de la uretrocistopexia suprapúbica por la técnica abierta, la técnica laparoscópica ofrece las ventajas señaladas en los estudios mencionados, ganando experiencia institucional suficiente para la comparación adecuada de ambos abordajes en estudios posteriores.

El interés del presente estudio de fomentar el aprendizaje de la técnica y de ganar experiencia institucional en la realización de la misma llevó a preferir las pacientes más delgadas y sin cirugía anterior para el grupo de cirugía por laporoscopia, motivo por el cual no se realizó el abordaje en pacientes con IMC mayores de 30 .

Ninguna de las pacientes en el estudio presentó persistencia de la incontinencia urinaria de esfuerzo. La aparición de incontinencia de urgencia es una complicación frecuente en los primeros meses post-operatorios de cirugía de incontinencia. La tasa de éxito encontrada $(94.2 \%)$ no tienen diferencia estadísticamente significativa. Sin embargo, el corto tiempo de seguimiento no permite establecer la verdadera tasa de éxito, ya que la mayoría de fracasos suceden en cualquier momento entre el mes 6 y 23 años post operatorios, según lo establecieron Spencer y O'Conor (16). Además, la tasa de éxito de la uretrocistopexia por laparoscopia parece caer considerablemente luego de un año (19).

El tiempo quirúrgico es significativamente mayor cuando se realiza el abordaje por laparoscopia y el hallado en el estudio se encuentra dentro de los promedios reportados en la literatura $(8-10,17,21)$, donde se menciona el efecto que ejerce la curva de aprendizaje sobre el tiempo quirúrgico total, siendo este menor a medida que se adquiere experiencia en la técnica (21). Otro factor que influye sobre el tiempo quirúrgico total es la presencia de complicaciones, que solucionadas bien 
por laparotomía o laparoscopia, incrementan de manera significativa esta variable.

Varios estudios mencionan que la técnica por laparoscopia disminuye el sangrado intraoperatorio comparada con la técnica abierta $(8-10,12)$. En el presente estudio no se evidenció una diferencia significativa en la variación de la concentración de Hemoglobina. Ninguna paciente requirió transfusión sanguínea, a pesar de que una paciente fue llevada a laparotomía por sangrado.

La frecuencia de complicaciones intraoperatorias fue alta comparada con estudios similares. La lesión vesical intra-operatoria es la complicación más frecuente reportada en la literatura. Liu y Paek (17) reportan cuatro lesiones vesicales en 107 procedimientos y Lam y Jenkins una en 58 procedimientos (10). Estos mismos autores mencionan que la frecuencia de las complicaciones tiende a disminuir a medida que progresan las series de casos, circunstancia que es previsible que suceda en nuestro medio a medida que se gane experiencia en la realización de la técnica por laparoscopia.

Las complicaciones post-operatorias y durante el seguimiento no se alejan de las reportadas en la literatura (18) siendo las más frecuentes las infecciones de vías urinarias, la retención urinaria, los hematomas del sitio quirúrgico y como ya se mencionó, la aparición de sintomatología de urgencia urinaria. En esta serie de casos no se presentaron hematomas.

El dolor post operatorio fue menor; hecho que se refleja en la significancia reducción de la cantidad de analgésico requerido en forma subjetiva por las pacientes. Muchos factores pueden influir sobre este resultado; el umbral de dolor de cada paciente, su nivel cultural, las expectativas de la paciente en cuanto a su tratamiento, la ansiedad, etc. Este hallazgo se correlaciona con los reportes de la literatura que mencionan como ventaja del abordaje laparoscópico, un menor dolor post-operatorio, menor requerimiento analgésico y más pronta reincorporación a la actividad diaria.

La estancia hospitalaria fue de 3.5 días, que contrasta con los reportes de la literatura, donde se reporta una disminución importante en la estancia hospitalaria. Esta circunstancia es explicable por el número de complicaciones intraoperatorias que requirieron una mayor vigilancia y cuidados intrahospitalarios.

El tamaño limitado de la muestra, el corto tiempo de seguimiento y el hecho de que se trata de la experiencia inicial con una nueva técnica es sin duda, una limitante importante en el presente estudio para establecer las ventajas reales del abordaje laparoscópico de la uretrocistopexia suprapúbica tipo Burch. Si bien a corto plazo el abordaje laparoscópico parece tener una tasa de éxito similar al abordaje abierto y hay menor dolor en el postoperatorio, las demás ventajas señaladas de menor morbilidad y disminución de estancia hospitalaria no se hicieron evidentes en el presente estudio, por las circunstancias antes mencionadas. En consecuencia, debe iniciarse un nuevo estudio, estudio ya controlado y aleatorizado con seguimiento a largo plazo, aprovechando las experiencias de esta fase piloto preliminar, destinado a establecer la efectividad, ventajas y conveniencia del abordaje laparoscópico, desde el punto de vista médico, tecnológico y de costos, ajustados a nuestro medio. Resultados preliminares de otros estudios parecen demostrar que el abordaje laparoscópico si es mejor costo-efectivo que la vía abdominal en lugares con mayor experiencia $(20)$.

Los resultados obtenidos resaltan la dificultad técnica del abordaje laparoscópico, que en manos expertas tiene una morbilidad alta por lo menos al comenzar a realizar la técnica. Esto pone de manifiesto la obligatoriedad de un entrenamiento adecuado y concienzudo para quienes desean realizar este abordaje.

\section{BIBLIOGRAFIA}

1. Cardozo L, Stanton SL. Genuine Stress Incontinence and detrusor instability: a review of 200 patients. Br J Obstet Gynaecol 1980; 87: 184-187.

2. Thompson JD, Wall LL, Growdon WA, Ridley JH. Incontinencia Urinaria de Esfuerzo En: Te Linde Ginecología Quirúrgica. Eds JD Thompson y JA Rock Editorial Médica Panamericana. Buenos Aires, 1993.

3. Burch JC. Urethrovaginal fixation to Cooper's Ligament for correction of stress Incontinence, cystocele and prolapse. Am J Obstet Gynecol 1961; 81:211-217.

4. Vancaille T, Schuessler W. Laparoscopic bladder neck suspension. J Laparo-endosc Surg 1991; 1 (3).

5. Feyereisl J, Dreherb F, Haenagi W, Zikmund J, Schneider H. Long Term results after Burch colposuspension. Am J Obstet Gynecol 1994: 171(3): 647-652.

6. Lomanto A, Sanchez J. Clínica de Incontinencia Urinaria. Instituto Materno Infantil. (Comunicación personal) 1993.

7. Dorsey JH, Cundiff G. Laparoscopic Procedures for incontinence and prolapse. Curr Opin Obstet Gynecol 1994; 6(3): 223-230.

8. Mc Dougall E, Klutke C, Cornell T. Comparison of transvaginal vs. Laparoscopic bladder neck suspension for stress urinary incontinence. Urology 1995; 45(4): 641-646.

9. Polascik T, Moore R, Rosemberg M, Kavoussi L. Comparison of laparoscopic and open retropubic urethropexy for treatment of stress urinary incontinence. Urology 1995; 45(4): 647-652.

10. Lam A, Jenkins G, Hyslop R. Laparoscopic Burch colposuspension for stress incontinence: preliminary results. Med J Aust 1995; 162(2): 18-22.
11. Rois P, Reisner G, Laporoscopic bladder Neck Suspension. Br J Urol 4: 1995.

12. Liu C. Laparoscopic retropubic colposuspension (Burch Procedure), A review of 58 cases. J Reprod Med 1993; 38(7): 526-530.

13. Riza E, Deshmukh A. A laparoscopic assisted extraperitoneal bladder neck suspension. A initial experience. J laparo-endosc Surg 1994; 4(5): $319-324$.

14. Jones J, Thompson R, Benrubi G. Suture Placement for hemostasis during laparoscopy using the Stamey needle. J Am Ass Gynecol Laparosc 1994; 2(4): 445-447.

15. Henley C. The Henley Staple-suture technique for laparoscopic burch colposuspension. J Am Ass Gynecol Laparosc 1994; 2(4): 441 447.

16. Spencer JR, O'Conor VJ. Comparison of procedures for stress urinary incontinence. AUA Update Series 6 (No. 28): 1987.

17. Liu CY, Paek W. Laparoscopic retropubic colposuspension (Burch procedure). Gynecol Laparoscop 1993: 1: 31-34.

18. Galloway NTM, Davies N, Stephenson TP: The complications of colposuspentions. Br J Urol 1987; 60: 122.

19. Lobel RW, Davis GD. Long-term results of laparoscopic Burch urethropexy. J Am Assoc Gynecol Laparoscop 1997: 4(3): 341-345.

20. Kung RC, Lie K, Lee P, Drutz HP. The cost-effectiveness of laparoscopic versus abdominal Burch procedures in woman with urinary stress incontinence. J Am Assoc Gynecol Laparoscop 1996 3(4): $537-544$

21. Radomski SB, Herschorn S. Laparoscopic burch bladder neck suspension: early results. J Urol 1996; 155: 515-518. 